\title{
Manifestaciones orales y caries dental en niños expuestos al virus de inmunodeficiencia humana
}

\section{Oral manifestations and dental caries in children exposed to human immunodeficiency virus}

\author{
María E. Dávila y Maritza Gil
}

Departamento de Medicina Preventiva y Social, Escuela de Medicina, Sección de Epidemiología y Bioestadística. Universidad Centroccidental Lisandro Alvarado. Barquisimeto, Venezuela. mangue98@hotmail.com,mdavila@ucla.edu.ve,mgil@ucla.edu.ve

Recibido 24 Enero 2011 /Enviado para Modificación 3 Octubre 2011/Aceptado 25 Octubre 2011

\section{RESUMEN}

Objetivo Determinar las manifestaciones orales y caries dental en niños expuestos al virus de inmunodeficiencia humana $(\mathrm{VIH})$.

Métodos Se estudiaron 37 niños que acuden a la consulta pediátrica del programa nacional de control de SIDA (PRONASIDA-Lara). Se informó a padres y representantes sobre los objetivos del estudio y su carácter confidencial. El examen de la cavidad oral lo realizó un odontólogo, quien además indagó sobre historia de manifestaciones bucales y corroboró la información con la historia clínica.

Resultado La edad promedio fue $5,15 \pm 3,79$ años, $62,2 \%$ era del género masculino. Se infectaron por trasmisión vertical $91,8 \%$. La mayoría de los niños pertenecían a la categoría clínica A $(35,1 \%)$. El mayor porcentaje $(37,8 \%)$ de niños se encontró en la categoría 15-24\% de linfocitos CD4. Entre las manifestaciones orales más prevalentes se encontró la candidiasis oral, queilitis angular, eritema gingival lineal y parotiditis. Se observó diferencia estadísticamente significativa entre el porcentaje de linfocitos CD4 y queilitis angular $(p<0,05)$. Cuarenta y cinco coma nueve (45,9\%) presentó caries dental y $29,7 \%$ tenían necesidad de tratamiento dental "urgente". Por otra parte, el índice de necesidad de tratamiento calculado (INT) muestra que tres cuartos de los niños tenían necesidad de tratamiento dental.

Conclusiones Los resultados del estudio permiten concluir que existe la necesidad de una estrategia basada en el manejo de las manifestaciones orales, frecuentes en la infección por VIH en niños, que permita un tratamiento desde un punto de vista integral, con un enfoque clínico y preventivo.

Palabras Claves Niños, VIH, manifestaciones bucales, caries dental (Fuente: DeCS, BIREME). 


\section{ABSTRACT}

Objective Determining oral manifestations and dental caries amongst children exposed to the immunodeficiency virus (HIV).

Methods Thirty-seven $(n=37)$ children attending the Colombian AIDS Control Program (PRONASIDA-Lara) pediatric service were studied. Parents and guardians were informed about the purpose of the study and its confidentiality. An oral examination was conducted by a dentist; parents/guardians were asked about their children's history of oral manifestations and this was corroborated with their clinical charts.

Results The mean age was $5.15 \pm 3.79 ; 62.2 \%$ were males. Ninety-eight point eight percent had become vertically infected. Most of the children were classified in the A immune suppression category $(35.1 \%)$. The highest percentage $(37.8 \%)$ of children were in the $15 \%-24 \%$ total CD4+ lymphocyte category. The most prevalent oral manifestations were oral candidiasis, angular cheilitis, linear gingival erythema and parotid enlargement. There was a significant statistical difference between percentages of CD4 lymphocytes and angular qualities $(p<0.05)$. Forty-five point nine percent had dental caries and $54.1 \%$ were in "urgent" need of dental treatment. The Index of Orthodontic Treatment Need (IOTN) showed that $3 / 4$ of the children needed dental treatment.

Conclusions The results of the study led to concluding that there was a need for an oral manifestation management-based strategy focusing on clinical and preventative treatment, as oral manifestations are frequent in children suffering from HIV infection.

Key Words: Children, HIV, oral manifestation, dental caries (source: MeSH, NLM).

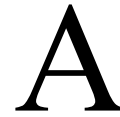

ún cuando la infección por el virus de inmunodeficiencia humana (VIH) en la última década ha experimentado un descenso, en algunos países persisten grupos de riesgo donde su prevalencia es alta. A nivel mundial existen 33,4 millones de personas que viven con VIH/SIDA (31,3 millones de adultos y 2,1 millones de menores de 15 años de edad). Para el 2008, el número estimado de nuevas infecciones en menores de 15 años de edad, fue de 430.000, cifra que representa un $18 \%$ menos que los casos reportados en el 2001. En Latinoamérica el número de casos reportados es de aproximadamente 2 millones y de ellos 6900 correspondía a menores de 15 años (1).

En su mayoría, los niños adquieren la infección en forma vertical (30-40 \%) y en aquellos a quienes no se les ha protegido con los protocolos de prevención, una vez infectados, la enfermedad suele progresar rápidamente hacia el SIDA (2). 
La clasificación pediátrica de la infección por VIH toma en consideración las categorías inmunológicas propuestas por el Centro para el Control y Prevención de Enfermedades de Atlanta (CDC) (3), considerando tres clases según la severidad de la inmunosupresión atribuible a la infección por el VIH, basada en el contaje de Linfocitos CD4 cuyas variaciones porcentuales son un buen indicador de la progresión de la enfermedad.

Por otra parte, también pueden ser clasificados en cuatro categorías clínicas, considerando los signos, síntomas o diagnóstico relacionado con la infección por VIH (3).

En las últimas décadas, el uso de antirretrovirales ha convertido a la infección por el virus VIH en una enfermedad crónica, tanto en niños como en adultos, prolongando y mejorando su calidad de vida (4).

La infección por VIH se caracteriza por la aparición de un conjunto de enfermedades consideradas como indicadores de la misma, entre las que se encuentran manifestaciones orales tales como la candidiasis oral, queilitis angular, leucoplasia vellosa, herpes simple, eritema gingival lineal y parotiditis, entre otras.

Numerosos estudios (5-11) han considerado las manifestaciones orales según su importancia para la predicción de la progresión de la enfermedad en pacientes pediátricos, encontrando que las lesiones orales como candidiasis oral, son las más prevalentes (21-67\%), seguidas de parotiditis $(2-4 \%)$, herpes simple (2-3\%) y eritema gingival lineal (4\%). Es por ello, que se hace necesario el diagnóstico temprano y tratamiento de las manifestaciones orales, así como el tratamiento de la caries dental, altamente prevalente según reportan la mayoría de los estudios (11-14), debido a la presencia de xerostomía, ingestión de antirretrovirales ricos en sacarosa, aunado a una pobre higiene oral.

La revisión de la información existente nos llevó a realizar una investigación para caracterizar a los niños expuestos al VIH según las manifestaciones orales y la caries dental, atendidos en la consulta del programa nacional VIH/SIDA del Estado Lara (PRONASIDA-Lara). Asimismo, la importancia del estudio radica en que no se encontró ningún otro publicado en la región, que caracterice a esta población desde un punto de vista epidemiológico. Los resultados evidencian la importancia de integrar el cuidado de la cavidad oral dentro del manejo clínico de los niños con infección por VIH, así como 
utilizar las manifestaciones orales más frecuentes como predictores de la progresión de la enfermedad, para adecuar su manejo y tratamiento.

\section{MÉTODOS}

Se realizó un estudio descriptivo en una muestra no-probabilística accidental conformada por 37 niños expuestos al virus de inmunodeficiencia humana, atendidos en la consulta pediátrica del Pronasida-Lara, en Barquisimeto, estado Lara, durante Mayo-Septiembre 2010, con el propósito de determinar las manifestaciones orales y caries dental, además de las variables demográficas (edad, genero); modo de trasmisión; categorías clínicas e inmunológicas; y algunos aspectos odontológicos generales de importancia. Se les informó a los padres y representantes, antes de iniciar la investigación, sobre el propósito del estudio; quienes accedieron a que sus hijos fueran examinados y firmaron voluntariamente la carta de consentimiento, fueron incluidos en el mismo.

Para la clasificación diagnóstica se utilizaron tres categorías clínicas basadas en los signos, síntomas o diagnósticos relacionados con la infección por VIH: A: levemente sintomático; B: moderadamente sintomático; C: severamente sintomático. Además se utilizaron las categorías inmunológicas según edad, basadas en el contaje de linfocitos T (CD4) y porcentaje total de linfocitos: sin inmunosupresión ( $\geq 25 \%$ ); supresión moderada (15-24 \%); supresión severa $(<15 \%)$, desarrolladas por el Centro para el Control y Prevención de Enfermedades de Atlanta (CDC) (3). Los niños expuestos al virus de inmunodeficiencia humana y que aún no han sido clasificados, se consideran en observación.

El examen de la cavidad oral lo realizó un odontólogo determinando la condición de los tejidos blandos y duros de la cavidad bucal y la necesidad de tratamiento dental.

Para el diagnóstico de la caries dental como indicador de salud bucal, se consideró "Diente cariado sin tratar" la pieza dentaría con al menos un área erosionada de aproximadamente $0.5 \mathrm{~mm}$ de diámetro. Igualmente se determinó la "Necesidad de tratamiento" considerándose tres categorías; a. Mantenimiento: cuando no exista dolor dentario, diente cariado sin tratar, lesiones orales, signos gingivales severos y no exista enfermedad periodontal establecida; b. No-urgente: cuando no exista dolor en la cavidad bucal, diente cariado que no comprometa la pulpa dentaria, restauraciones fracturadas sin presencia de caries dental, sin signos gingivales y sin enfermedad periodontal; 
c. Urgente: cuando exista dolor dentario, caries dental que comprometa la pulpa dentaria y fractura de restauraciones o pérdida de la restauración (15).

También se calculó el Índice de Necesidad de Tratamiento (INT) utilizando la formula que consiste en dividir el total de dientes con caries dental entre el total de dientes con caries dental más dientes obturados por 100 (16).

Se interrogó a los padres y/o representantes sobre historia de manifestaciones orales tales como candidiasis oral, herpes simple, parotiditis, entre otras y se corroboró con lo asentado en la historia clínica.

Para el procesamiento estadístico de las variables se utilizó el paquete estadístico SPSS Versión 15.0. Para el análisis se empleó estadística descriptiva y pruebas no paramétricas para comparar los grupos (Test de Kruskal Wallis).

\section{RESULTADOS}

Se realizó el análisis descriptivo de las variables sociodemográficas de 37 niños expuestos al VIH. La edad promedio fue de 5,13 $\pm 3,79$ años, la mayoría de ellos se encontraba en el grupo de 6-12 años (48,6\%), seguido por los menores de 12 meses $(29,7 \%)$. El 62,2 \% eran del género masculino $(\mathrm{n}=23)$. El modo de trasmisión más frecuente fue la vertical (91,5\%), (datos no presentados).

Los estadios clínicos más frecuentes fue el $\mathrm{A}(35,1 \%)$, seguido por el estadio C (13,5\%). Con relación al contaje porcentual de linfocitos CD4, ocho niños $(21,6 \%)$ presentaban inmunosupresión severa $(<15 \%)$ (Tabla 1$)$.

Tabla 1. Categorización Clínica e Inmunológica de los niños expuestos al síndrome de inmunodeficiencia humana según CDC

\begin{tabular}{ccc}
\multicolumn{2}{c}{ al síndrome de inmunodeficiencia humana según CDC } \\
\hline Clínica $^{\mathrm{a}}$ & $\mathrm{N}^{\circ}$ & $\%$ \\
\hline A & 13 & 35,1 \\
B & 6 & 16,2 \\
C & 5 & 13,5 \\
En observación & 13 & 35,1 \\
Imunológica & & \\
\% Linfócitos CD4 & \\
$<15 \%$ & 8 & 21,6 \\
$15-24 \%$ & 14 & 37,8 \\
$>25 \%$ & 2 & 5,4 \\
\hline${ }^{\mathrm{a}} \mathrm{n}=37 ;{ }^{\mathrm{b}} \mathrm{n}=24$. Se excluyeron del análisis los niños en observación $(\mathrm{n}=13)$
\end{tabular}


La principal manifestación oral encontrada fue la candidiasis oral $(73,0 \%)$, seguida de eritema gingival lineal $(27,9 \%)$ y queilitis $(24,3 \%)$. Entre otras manifestaciones clínicas de interés la parotiditis representó $24,3 \%$ (Tabla 2).

La Tabla 3 muestra la presencia de caries dental en 45,9 \% de los niños. Cuarenta y cuatro coma cuatro por ciento $(44,4 \%)$ de los mayores de 6 años presentaban caries en el primer molar permanente. Al estudiar la necesidad de tratamiento se observó que $29,17 \%$ requerían tratamiento dental urgente por causas como: dolor dentario; caries con compromiso pulpar; fractura o pérdida de obturaciones. El Índice de Necesidad de Tratamiento (INT) reveló que 75 \% de los niños necesitaba de tratamiento dental.

Tabla 2. Manifestaciones orales en niños expuestos al virus de inmunodeficiencia humana

\begin{tabular}{|c|c|c|}
\hline Manifestaciones Orales $^{a}$ & $\mathrm{~N}^{\circ}$ & $\%$ \\
\hline \multicolumn{3}{|c|}{ Candidiasis oral } \\
\hline SI & 10 & 73,0 \\
\hline NO & 27 & 27,0 \\
\hline \multicolumn{3}{|c|}{ Queilitis angular } \\
\hline SI & 9 & 24,3 \\
\hline NO & 28 & 75,7 \\
\hline \multicolumn{3}{|c|}{ Eritema gingival lineal } \\
\hline SI & 10 & 27,0 \\
\hline NO & 27 & 73,0 \\
\hline \multicolumn{3}{|c|}{ Parotiditis } \\
\hline SI & 9 & 24,3 \\
\hline NO & 28 & 75,7 \\
\hline
\end{tabular}

Tabla 3. Caries dental, necesidad de tratamiento e índice de necesidad de tratamiento (INT) a en los niños expuestos al virus de inmunodeficiencia humana

\begin{tabular}{|c|c|c|}
\hline Item & $\mathrm{N}^{\circ}$ & $\%$ \\
\hline \multicolumn{3}{|l|}{ Caries dental ${ }^{b}$} \\
\hline $\mathrm{Si}$ & 17 & 45,9 \\
\hline No & 20 & 54,1 \\
\hline \multicolumn{3}{|c|}{ Caries $1 \mathrm{er}$ molar permanente ${ }^{c}$} \\
\hline $\mathrm{SI}$ & 8 & 44,4 \\
\hline No & 10 & 56,6 \\
\hline \multicolumn{3}{|c|}{ Necesidad de tratamiento $^{d}$} \\
\hline Mantenimiento & 20 & 54,1 \\
\hline No Urgente & 6 & 16,2 \\
\hline Urgente & 11 & 29,7 \\
\hline
\end{tabular}

$\mathrm{Al}$ relacionar la categoría diagnóstica clínica con las manifestaciones orales, destaca que más del $30 \%$ de los niños en las categorías "A y C" presentaban candidiasis oral, queilitis angular y herpes simple. El eritema gingival lineal predominó en los niños de la categoría "A". Se observó igual porcentaje de 
parotiditis $(12,5 \%)$ en las tres categorías clínicas (Tabla 4). No se observó diferencia estadísticamente significativa $(\mathrm{p}>0,05)$ entre las categorías clínicas $\mathrm{y}$ las diferentes manifestaciones orales (datos no presentados).

Tabla 4. Manifestaciones orales en los niños expuestos al virus de inmunodeficiencia humana según categoría clínica $(C D C)^{a}$

\begin{tabular}{|c|c|c|c|c|}
\hline \multirow{2}{*}{$\begin{array}{c}\text { Categoría } \\
\text { Diagnóstica }\end{array}$} & \multicolumn{2}{|c|}{ SI } & \multicolumn{2}{|c|}{ NO } \\
\hline & $\mathrm{N}^{\circ}$ & $\%$ & $\mathrm{~N}^{\circ}$ & $\%$ \\
\hline \multicolumn{5}{|c|}{ Candidiasis Oral } \\
\hline$A$ & 5 & 50,0 & 8 & 57,1 \\
\hline B & 2 & 20,0 & 4 & 28,6 \\
\hline C & 3 & 30,0 & 2 & 14,3 \\
\hline \multicolumn{5}{|c|}{ Queilitis Angular } \\
\hline A & 4 & 44,4 & 9 & 60,0 \\
\hline$B$ & 2 & 22,2 & 4 & 26,7 \\
\hline C & 3 & 33,3 & 2 & 13,3 \\
\hline \multicolumn{5}{|c|}{ Herpes Simple } \\
\hline$A$ & 2 & 40,0 & 11 & 57,9 \\
\hline B & 1 & 20,0 & 5 & 26,3 \\
\hline C & 2 & 40,0 & 3 & 15,8 \\
\hline \multicolumn{5}{|c|}{ Eritema Gingival Lineal } \\
\hline$A$ & 5 & 20,8 & 83 & 3,3 \\
\hline B & 2 & 8,3 & 41 & 6,6 \\
\hline C & 2 & 8,3 & 31 & 2,5 \\
\hline \multicolumn{5}{|c|}{ Parotiditis } \\
\hline A & 3 & 12,5 & 10 & 41,6 \\
\hline$B$ & 3 & 12,5 & 3 & 12,5 \\
\hline C & 3 & 12,5 & 2 & 8,3 \\
\hline
\end{tabular}

Tabla 5. Manifestaciones orales en los niños expuestos al virus de inmunodeficiencia humana según porcentaje células de linfocitos CD4 ${ }^{\text {a }}$

\begin{tabular}{|c|c|c|c|c|c|c|c|c|c|c|c|}
\hline \multirow{4}{*}{ Manifestaciones orales } & \multirow{2}{*}{\multicolumn{4}{|c|}{$>25$}} & \multirow{2}{*}{\multicolumn{3}{|c|}{$\begin{array}{c}\text { \% linfocitos CD4 } \\
15-24\end{array}$}} & \multirow{2}{*}{\multicolumn{4}{|c|}{$<15$}} \\
\hline & & & & & & & & & & & \\
\hline & \multicolumn{2}{|r|}{$\mathrm{Si}$} & \multicolumn{2}{|r|}{ No } & $\mathrm{Si}$ & \multicolumn{2}{|c|}{ No } & \multicolumn{2}{|r|}{$\mathrm{Si}$} & \multicolumn{2}{|c|}{ No } \\
\hline & $\mathrm{N}^{\circ}$ & $\%$ & & No $\%$ & No $\%$ & & $\%$ & $\mathrm{~N}^{\circ}$ & $\%$ & & jo $\%$ \\
\hline Candidiasis oral & 2 & 100,0 & - & - & $4 \quad 28,5$ & 10 & 71,5 & 4 & 50,0 & 4 & 50,0 \\
\hline Queilitis angular ${ }^{\mathrm{b}}$ & 2 & 100,0 & - & - & 214,3 & 12 & 85,7 & 5 & 62,5 & 3 & 37,5 \\
\hline Herpes simple & 1 & 50,0 & 1 & 50,0 & 17,14 & 13 & 92,8 & 3 & 37,5 & 5 & 62,5 \\
\hline Eritema gingival & 1 & 50,0 & 1 & 50,0 & 428,6 & 10 & 71,4 & 4 & 50,0 & 4 & 50,0 \\
\hline Parotiditis & 1 & 50,0 & 1 & 50,0 & $4 \quad 28,6$ & 10 & 071,4 & 4 & 50,0 & 4 & 50,0 \\
\hline
\end{tabular}

La Tabla 5, muestra los porcentajes obtenidos al relacionar las manifestaciones orales con las categorías inmunológicas. En la categoría $<15$ $\%$ (inmunosupresión severa) $62,5 \%$ de los niños habían sufrido de queilitis 
angular. Mientras que candidiasis oral, eritema gingival lineal y parotiditis la sufrieron 50,0 \% de los niños. En la categoría 15-24 \% (inmunosupresión moderada) al menos $28,6 \%$ presentaron alguna de las manifestaciones orales.

\section{DISCUSIÓN}

La infección por VIH en algunos países ha ido en descenso, sin embargo aun persisten grupos de riesgo en los que se encuentran los niños.

Estudios revelan que la principal forma de contagio es la transmisión vertical (1-4,8), en esta investigación el modo de transmisión vertical represento 91,5\%.

Por otra parte, se consideró la clasificación clínica e inmunológica desarrollada por el CDC (3), para categorizar a los niños, los resultados obtenidos son menores a los encontrados por otros autores $(2,10,14,20)$. De los 37 niños examinados, $35,1 \%$ y $16,2 \%$ se encontraban en la clasificación A y B. Trece coma cinco por ciento $(13,5 \%)$ se encontraba en la categoría de inmunosupresión severa y $35,1 \%$ en observación.

Igualmente, en la categorización por contaje de linfocitos CD4, 21,6\% de los niños se encontraba en la categoría inferior al $15 \%$ (inmunosupresión severa), 37,8 \% en la categoría 15-24\% (inmunosupresion moderada), y en la categoría mayor a $25 \%$ (no supresión) $5,4 \%$, estos valores son comparables a los reportados en otras investigaciones $(10,14,19)$.

Las manifestaciones orales en la mayoría de los casos de infección pediátrica por VIH, aparecen como los primeros signos de la enfermedad con una alta prevalencia. Como ha sido reportado por algunos investigadores $(6,7,11,17,18,21)$, las manifestaciones orales no solo pueden ser indicadores de la infección por el VIH, sino que pueden ser consideradas como marcadores clínicos de la infección, llegando a predecir su progreso al SIDA.

Entre las manifestaciones orales se encuentra la candidiasis oral considerada en algunos países (5) como la más prevalente de las lesiones orales en niños $(67 \%)$, seguido del herpes simple, parotiditis, y eritema gingival lineal $(14,17,19,21)$. En la presente investigación, se observó que $27 \%$ de los niños habían sufrido de candidiasis oral, esta cifra es similar a la reportada en algunos estudios $(9,19)$ y menor a la encontrada por Benito y colaboradores (10) y Soto y colaboradores (14) en otras regiones de Venezuela. El eritema gingival lineal, ha sido asociado con la infección por VIH y considerado como 
una de las manifestaciones orales más comunes $(19,21)$, los resultados obtenidos demuestran que $27 \%$ de los niños la presentaban, este resultado es inferior al reportado en otros autores $(10,21)$.

Por otra parte, las manifestaciones orales tales como la queilitis angular y la parotiditis la presentaron $24,3 \%$ de los niños, este resultado es menor al reportado por Solis y colaboradores (7) y mayor al encontrado en otros estudios $(5,11,14)$. El herpes simple fue la manifestación oral menos frecuente (16,2\%) pudiéndose comparar los resultados con la mayoría de los reportados por otros investigadores $(9,14,17,19,20)$.

Es importante resaltar a la luz de los resultados de la presente investigación, que en su mayoría los niños inmunosuprimidos o no, presentaron en algunas manifestaciones orales. Los resultados del estudio muestran para todas las categorías inmunológicas (\% linfocitos CD4) que las manifestaciones orales más frecuentes fueron la candidiasis oral, queilitis angular, eritema gingival y la parotiditis, similar a los hallazgos reportados en otros estudios $(5,14,19,21)$.

$\mathrm{Al}$ indagar sobre la relación entre la categoría inmunológica y las presencia de manifestaciones orales, se observó diferencia estadísticamente significativa ( $p>0.05$ ) entre la categoría inmunológica y la queilitis angular, sin embargo es en el estadio A y $\mathrm{C}$ donde se observó el mayor porcentaje de manifestaciones orales. Por otra parte, las manifestaciones orales fueron más prevalentes en las categorías inmunológicas inferiores al $15 \%$. Los resultados obtenidos corroboran los encontrados en otras investigaciones $(10,14,19)$.

Existen controversias entre la susceptibilidad a la caries dental en los niños con infección por VIH, algunos opinan que no existen diferencias de importancia mientras que otros reportan una alta prevalencia (5). En tal sentido, en el estudio se encontró un alto porcentaje de niños sin caries $(54,1 \%)$, este resultado es comparable con el reportado por Hicks y colaboradores (13), y mayor al encontrado por Fachin y colaboradores (14). El porcentaje de niños con caries en el primer molar permanente fue de $44,4 \%$.

Con relación a la necesidad de tratamiento dental, aproximadamente $30 \%$ de los niños requerían de tratamiento dental urgente, lo que podría ser un indicador de una higiene bucal deficiente $(5,8)$. Por otra parte el cálculo del INT reporta que $3 / 4$ de los niños presentaban necesidad de tratamiento dental. 
La deficiente higiene oral encontrada, junto con las manifestaciones orales asociadas a la enfermedad, aumentan el riesgo al deterioro la salud general de los niños. Se hace evidente el papel que juegan los profesionales de la odontología en la prevención, control, diagnóstico precoz y tratamiento de las manifestaciones orales en niños que viven con el VIH.

Es por ello, que es necesario a la luz de los hallazgos de la presente investigación, una estrategia general de tratamiento basada en el manejo de las manifestaciones orales, asociadas a la infección, desde un punto de vista integral tanto clínico como preventivo •

\section{REFERENCIAS}

1. UNAIDS. Uniting the world against Aids. Aids Epidemic update 2009. [Internet]. Disponible en: http://www.data.unaids.org/pub/Report/2009/2009_epidemic_update_en.pdf Consultado 18 Enero 2011.

2. Bustamante A, Elorza M, Cornejo N. Características Clínicas de niños infectados por VIH atendidos en un hospital Universitario de Medellín, Colombia 1997-2007. IATREIA. 2007; 2(4): 354-360.

3.1994 Revised Classification System for Human Immunodeficiency Virus. Infection in Children less than 13 years of age. MMWR. Center for disease control and prevention. Sept 30 1994. Vol 43 . RR-12.

4. Desafíos. La niñez y EI VIH/Sida en America Latina. [Internet]. Disponible en: http:// www.unicef.org/lac/Desafios7_esp(2).pdf Consultado: 22 de Enero 2011.

5. Coogan M, Greenspan J; Challocombe S. Oral lesions in infection with human immunodeficiency virus. Bulletin of the World Health Organization. sept 2005; 83(9):700-706.

6. Garcia R, Garay C, Hernandez F. Manifestaciones orales de la Infección del VIH/SIDA. Revista Medica Electrónica 2006; 28(6):1-8. [Internet] Disponible en: http:// www.revmatanzas.sld.cu/revista\%20medica/ano\%202006/vol6\%202006/ tema15.htm Consultado: 02 de Octubre 2010.

7. Solís G, Castillo M, Torres R. Manifestaciones orales en niños con VIH/SIDA hospitalizados en el servicio de infectología del instituto de salud del niño (Enero-Junio 1998). Folia dermatología Peruana. 2000; 11(2):1-8.

8. Marin ML, Hurtado PA, Santamaria LA, et al. Estado de salud bucal de niños con diagnóstico de VIH/SIDA en la Fundación Endes de Medellín. Presentación de casos y revisión de la Literatura. Rev Fac Odont Univ de Antioquia. 2004; 15(2):64-75.

9. Álvarez L, L; Hermida L, Cutiño E. Situación de Salud oral de los niños uruguayos portadores del virus de inmunodeficiencia humana. Arch Pediatr Urug. 2007; 78(1):1-10.

10. Benito M; Rojas I; Garcia R; Villalobos H, et al. Relación entre las manifestaciones orales asociadas al VIH-SIDA pediátrico y las categorías clínicas inmunológicas. Cienc odontol. ene-jun 2005;2(1):34-41.

11. Hodgson TA, Naidoo S, Chidzonga M, Ramos-Gómez F, Shiboski C. Identification of oral health care needs in children and adults, Management of oral diseases. Adv Dent Res. April 2006; 19:106-117.

12. Exposito D, AJ; Vallejo B, E; Martos C, EG. Manifestaciones orales de la infección por VIH en la infancia: articulo de revisión. Med Oral Patol Oral Cir Bucal. 2004; 9 (5) :410420. 
13. Hicks MJ, Flaitz CM, Carter AB. Dental caries in HIV-infected children: a longitudinal study. Pediatric Dentistry. 2000;22 (5): 359-364.

14. Soto de Fachin M; Casanova de Escalona L; Hoffmann I; Pugliese R. Lesiones Locales en niños VIH/SIDA y su relación con Linfocitos CD4 y carga Viral. Valencia. Arch Ven de Pueri y Pediat. 2007; 27(2):47-52.

15. White J, Beltrán ED, Perlman S. Training manual for standardized oral screening. Division of Oral Health. National Center for Chronic Disease and Health Promotion. Center for Disease Control and Prevention. Atlanta, revised Edition, Nov. 2004. [Internet]. Disponible en: http://www.media.specialolympics.org/soi/files/.../HA_SS_Training+Manual.pdf Consultado: 18 de Diciembre 2010.

16. Jon A. Dental public health community dentistry. St Louis: Mosby Co. 1981; p.74-88.

17. Ramos Ramos-Gómez FJ, Hilton JF, ConcholaAJ, Greespan D, Greenspan JS, Maldonado. YA. Risk factors for HIV -related orofacial soft-tissue manifestations in children. Pediatric Dent. 1999;18:121-126.

18. Ramos-Gómez FJ, Petru A, Hilton JF, Conchola AJ, Wara D, Greenspan JS. Oral manifestations and dental status in pediatric HIV infection. Int J Paediatric Dentistry. 2000;10;3-11.

19. Gaitan- Cepeda L, Cashat-Cruz M, Morales-Aguirre JJ, Sánchez-Vargas L, Aquino-Garcia $\mathrm{S}$, Fragoso-Rios $\mathrm{R}$, et al. Prevalence of oral lesions in Mexican children with perinatally acquired HIV: association with immunologic status, viral load, and gender. AIDS Patient Care STD 2000;16:151-156. [Internet]. Disponible en: http://www. bvssida.insp.mx/articulos/3163.pdf. Consultado: 19 de Noviembre 2010.

20. Ferreira Soares L, Barbosa de Araujo G, Pomarico Ribeiro de Sousa I. Pediatric HIVrelated oral manifestations -five year retrospective study. Braz Oral Res. 2004; 18(1) 6-11.

21. Howel RB, Jandinski JJ, Palumbo P, Shey Z, Houpt MI. Oral soft tissue manifestations and CD4 lymphocyte counts in HIV-infected children. Pediatric Dentistry. 1996, 18 (2): 117-120. 